Yuika Sasatani', Shinichiro Okauchi', Gen Ohara', Katsunori Kagohashi', Kesato Iguchi', Koji Kawai', Hiroaki Satoh ${ }^{1}$

'Mito Medical Center, University of Tsukuba, Miya-machi, Mito, Japan

${ }^{2}$ University of Tsukuba, Amakubo, Tsukuba, Japan

\title{
Oligo-recurrence from anaplastic lymphoma kinase-rearranged lung adenocarcinoma
}

\begin{abstract}
Anaplastic lymphoma kinase rearranged non-small-cell lung cancer is a rare disease. Among them, a subset of patients exist who exhibit relatively slowly progressing symptoms and have oligo-metastases. In this article, we present two cases of ALK rearranged lung adenocarcinoma in patients who experienced postoperative oligo-recurrence. Both cases were treated with surgical resection and gamma knife irradiation for oligo-recurrence. After local therapy, the first patient remained disease free for over 23 months; the second for over 18 months. It appears that some patients with ALK rearranged NSCLC experience oligo-recurrence in their clinical course. For such patients, appropriate local therapy may be beneficial in improving both the quality of life and the prognosis.
\end{abstract}

Key words: oligo-recurrence, local therapy, ALK rearrangement

Adv Respir Med. 2019; 87: 301-304

\section{Introduction}

In non-small-cell lung cancer (NSCLC), the understanding of the carcinogenic mechanism has progressed. In more than half of NSCLC patients, driver gene mutations have received increasingly more interest as the causative factor in the pathogenesis of this disease [1]. For them, it is now possible to administer drugs that can be expected to have a specific effect. ALK rearranged NSCLC is one type of NSCLC with known driver mutation genes, and it accounts for a small percentage of NSCLC patients [1]. However, even in the presence of drugs with effective specificity, the majority of patients who achieved good responses will still become victims of progressive disease. In particular, the prognosis for patients suffering from lung cancer with distant metastasis remains poor. Progress free survival time varies among patients and is unpredictable. Similarly, the sites of recurrence are also unpredictable at this time.
In this article, we report on two patients with solitary small metastatic lesions as a postoperative recurrence where local therapies were available. Both patients did not have rapid progress thereafter. The presence of ALK rearranged NSCLC patients who showed such unusual clinical courses is considered to be notable.

\section{Material and methods}

\section{Case 1}

A 43-year-old man was noted to have an abnormal opacity in chest radiograph during mass-screening. Chest CT scan revealed a nodule in the lower lobe of the right lung (Figure 1A). Hilar and mediastinal lymph node adenopathy was not observed. He was referred to our hospital for further examination and treatment. Physical examination and laboratory data were unremarkable. Right lower lobectomy with nodal dissection was performed, and the final pathological diagnosis was adenocarcinoma. Pathological tumor stage

Address for correspondence: Hiroaki Satoh, Division of Respiratory Medicine, Mito Medical Center, University of Tsukuba, Miya-machi 3-2-7, Mito-city, Ibaraki, 310-0015, Japan; e-mail: hirosato@md.tsukuba.ac.jp

DOI: 10.5603/ARM.2019.0053

Received: 17.06.2019

Copyright (C) 2019 PTChP

ISSN 2451-4934 
was proven to be T1aN0M0. Fluorescence in situ hybridization of the resected specimen revealed an ALK translocation. Thereafter, he was followed up at our outpatient department without any additional adjuvant therapy. Thirty-four months after the surgery, follow up chest CT scan revealed 4 new nodules up to $10 \mathrm{~mm}$ in size in the left lung. Two of them were detected in left $S^{6}$, and the others were in left $S^{5}$ and $S^{8}$ (Figure 1B). The patient had no symptoms and his PS was zero at this time. Brain MRI, bone scan and CT of the abdomen showed no other distant metastasis. These 4 nodules in the left lung were surgically removed, and 2 of them in left $S^{6}$ were pathologically confirmed to be metastatic lesions. He is still alive and well with no evidence of recurrence in PET/CT and brain MRI 23 months after the surgical resection of the oligo-recurrence.

\section{Case 2}

A 39-year-old man was admitted to our hospital due to an abnormal opacity in chest radiograph during mass-screening. Chest CT scan revealed a nodule in the lower lobe of the left lung (Figure 2A). Hilar and mediastinal lymph node adenopathy was not observed. He was referred to our division for further examination and treatment. Physical examination and laboratory data were unremarkable. Left pneumonectomy with nodal dissection was performed, and the final pathological diagnosis was adenocarcinoma. Pathological tumor stage was proven to be T1bN2M0. Fluorescence in situ hybridization of the resected specimen revealed an ALK translocation. He received 6 courses of adjuvant chemotherapy with cisplatin, pemetrexed and bevacizumab. Thereafter, the patient received maintenance chemotherapy with pemetrexed and bevacizumab. PET/CT scan taken 17 months after the pneumonectomy revealed a small nodule of the left adrenal grand. Morphological appearance and SUVmax value suggested adenoma of the adrenal gland. After follow up at our outpatient department, the nodule did not receive any additional adjuvant therapy. Twenty-four months after the pneumonectomy, three small nodules up to $10 \mathrm{~mm}$ in diameter were detected in a brain MRI (Figure 2B), which were treated with gamma knife. At the same time, the nodule of the left adrenal gland became enlarged with high FDG-uptake on PET/CT (Figure 2C), and it was resected surgically 27 months after the pneumonectomy. Pathologically, the nodule was confirmed to be a metastatic lesion. The patient started to receive crizotinib after the local therapies and is still alive and well with no evidence of recurrence in PET/CT and brain MRI 18 months after the surgical resection of the adrenal oligo-recurrence.

\section{Discussion}

ALK rearranged NSCLC accounts for $2-5 \%$ of all the different varieties of NSCLC, with adenocarcinoma being the most common histological type [2]. The response period is fairly long due to the specific effect of ALK-TKI, but almost all of the patients eventually experience recurrence. In this report, we identified and examined 2 patients
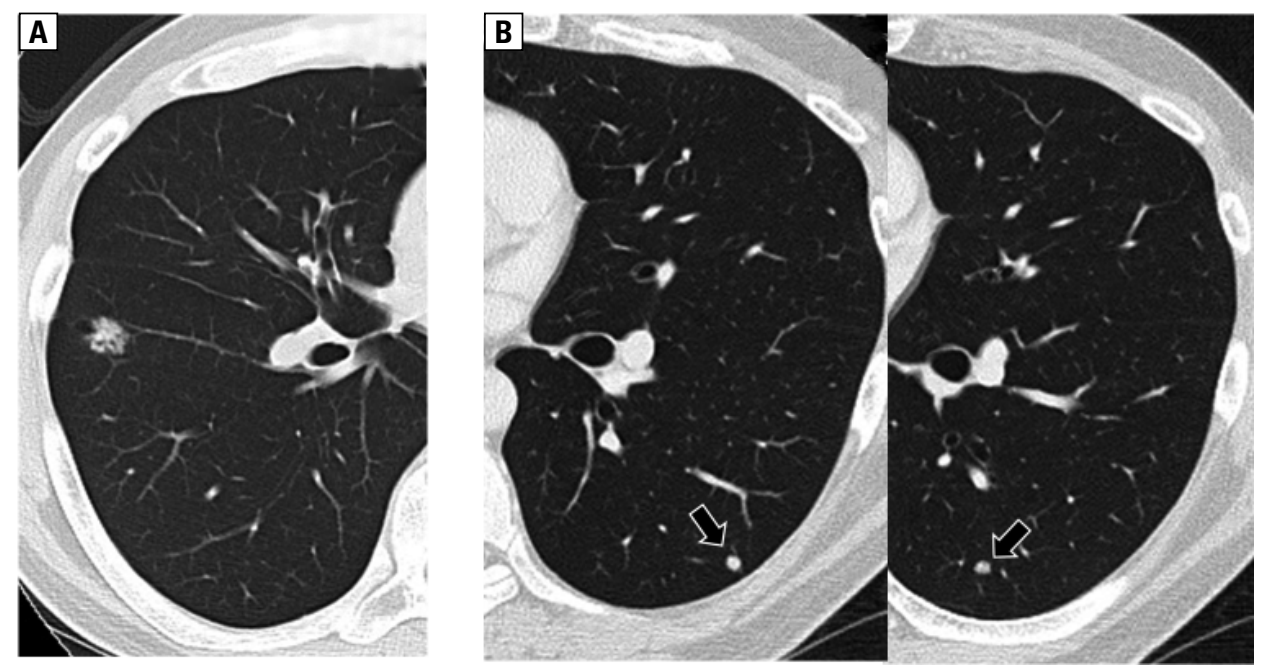

Figure 1. A. Chest CT scan of case 1 at the first visit, which showed a nodule in the right lower lobe. B. Chest CT scan of case 1 taken at 34 months after the right lower lobectomy. Two nodules detected in left $S^{6}$ (arrows) were confirmed as metastatic lesions pathologically 

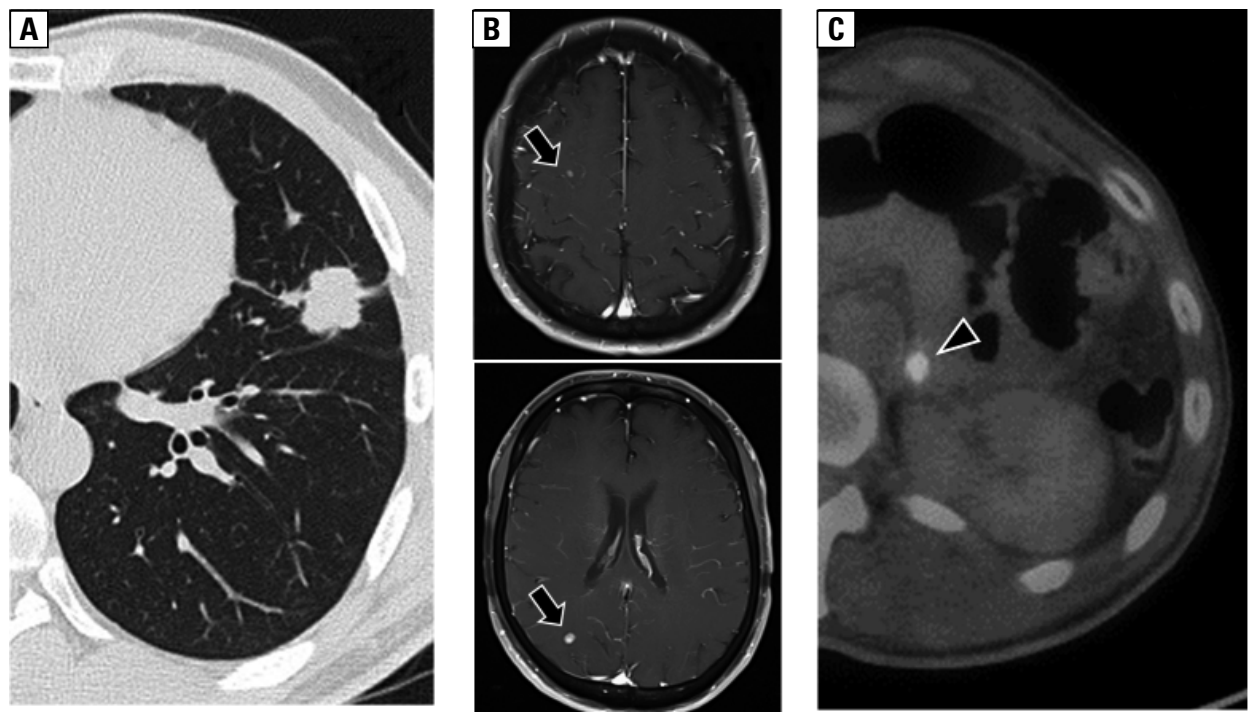

Figure 2. A. Chest CT scan of case 2 at the first visit, which showed a nodule in the left lower lobe. B. Brain MRI of case 2 taken at 24 months after the pneumonectomy. Three small nodules up to $10 \mathrm{~mm}$ in diameter were detected (arrows) and treated with gamma knife. C. FDG-PET/CT of case 2 taken at 24 months after the pneumonectomy. A nodule with high FDG-uptake (arrow head) was detected in the left adrenal gland and resected surgically

who presented oligo-recurrence during their long clinical courses, which were treated surgically or radiologically.

More than 20 years ago, Wechselbaum and Hellman proposed the term "oligometastases" [3], and thereafter, Niibe et al. proposed the concept of "oligo-recurrence" [4, 5]. According to them, oligometastases was defined as between one and several distant metastases/recurrences in one to several organs that can be treated by local therapy [3]. However, the patients who had oligometastases with an uncontrolled primary site suffered from treatment failure. The most important prognostic factor for oligometastases is the status of the primary site [4-6]. Niibe et al. proposed the new notion of oligo-recurrence to overcome these problems. Oligo-recurrence is a notion similar to oligometastases, but oligo-recurrence was defined as the condition with a controlled primary lesion $[4,5]$. In the states of oligometastases and oligo-recurrence, patients with 1-2 metastases and recurrences reportedly showed better prognosis than those with 3-5 metastases and recurrences [7].

When analyzing oligo-recurrence of NSCLC in only the brain or adrenal gland, patients achieved favorable survival [6, 8]. In order to discover oligo-recurrence, positron emission tomography is considered to be essential. Also, MRI is considered essential in order to detect oligo-recurrence to the brain. In fact, in both patients of this report, oligo metastatic sites were found by PET/CT and brain MRI. The patient had no subjective symptoms at the time of lesion detection.
In patients with ALK rearranged NSCLC, the initiation of ALK-TKI is one of the treatment options when oligo-recurrence is found. If it can be treated with local therapy, however, there may be an option to withhold initiation of ALK-TKI and follow up the clinical course for a while. In fact, in case 1, ALK-TKI was not administered and followed up, but no recurrence was confirmed by PET/CT and brain MRI even 23 months after resection of the oligo-recurrence. In case 2, oligo-recurrence to the brain and left adrenal grand developed in the period of PEM + BEV maintenance therapy after additional postoperative treatment. After local therapy with stereotactic brain irradiation to the brain and adrenalectomy, therapy with crizotinib was initiated. No recurrence was detected on PET/CT and brain MRI for 18 months after the local therapy. If ALK-TKI is administered, there is a high possibility of response, and long-term PFS will be obtained. However, the possibility of recurrence at any time remains high. Thus, in oligo-recurrence, the concept of using each effective treatment successfully over a long period of time may be important.

Control of the primary tumor, good nutritional status, less aggressive histological type and a small number of metastases are factors which impact the treatment of oligo-recurrence $[4,5$, $9,10]$. If local therapy is properly administered, oligo-recurrence allows for long-term control and avoids loss of quality of life. The detection of tiny oligo metastatic sites needs to be very carefully monitored, as oligo-recurrence may lead to long- 
-term survival. In view of the current technological advances and the use of local and systemic treatments, surgery $[4,5,9,10]$ is appropriate for some patients with oligo-recurrence. Radiation therapy is the next best treatment. Stereotactic radiosurgery is an effective treatment, especially for oligo-recurrence to the brain.

In summary, among the patients with ALK rearranged NSCLC, it appears that some patients develop oligo-recurrence in their long clinical courses. It is difficult to evaluate whether it is really oligo-recurrence. Regardless, for such patients, appropriate local therapy may be beneficial in improving the quality of life and prognosis. ALK rearranged NSCLC is a rare disease. In such rare diseases, information about oligo-recurrence is not available from clinical trials. Therefore, accumulating and sharing clinical experience is considered to be significant.

\section{Conflict of interest}

None declared.

\section{References:}

1. Griffin R, Ramirez RA. Molecular targets in non-small cell lung cancer. Ochsner J. 2017; 17(4): 388-392, indexed in Pubmed: 29230123.
2. Vestergaard HH, Christensen MR, Lassen UN. A systematic review of targeted agents for non-small cell lung cancer. Acta Oncol. 2018; 57(2): 176-186, doi: 10.1080/0284186X.2017.1404634, indexed in Pubmed: 29172833.

3. Weichselbaum RR, Hellman S. Oligometastases revisited. Nat Rev Clin Oncol. 2011; 8(6): 378-382, doi: 10.1038/nrclinonc.2011.44, indexed in Pubmed: 21423255.

4. Niibe Y, Chang JY, Onishi H, et al. Oligometastases/Oligo-recurrence of lung cancer. Pulm Med. 2013; 2013: 438236, doi: 10.1155/2013/438236, indexed in Pubmed: 23476762.

5. Niibe Y, Hayakawa K. Oligometastases and oligo-recurrence: the new era of cancer therapy. Jpn J Clin Oncol. 2010; 40(2): 107111, doi: 10.1093/jjco/hyp167, indexed in Pubmed: 20047860.

6. Lopez Guerra JL, Gomez D, Zhuang Y, et al. Prognostic impact of radiation therapy to the primary tumor in patients with non -small cell lung cancer and oligometastasis at diagnosis. Int J Radiat Oncol Biol Phys. 2012; 84(1): e61-e67, doi: 10.1016/j. ijrobp.2012.02.054, indexed in Pubmed: 22503522.

7. Salama JK, Chmura SJ, Mehta N, et al. An initial report of a radiation dose-escalation trial in patients with one to five sites of metastatic disease. Clin Cancer Res. 2008; 14(16): 5255-5259, doi: 10.1158/1078-0432.CCR-08-0358, indexed in Pubmed: 18698045.

8. Holy R, Piroth M, Pinkawa M, et al. Stereotactic body radiation therapy (SBRT) for treatment of adrenal gland metastases from non-small cell lung cancer. Strahlenther Onkol. 2011; 187(4): 245-251, doi: 10.1007/s00066-011-2192-z, indexed in Pubmed: 21424513

9. Jabbour SK, Daroui P, Moore D, et al. A novel paradigm in the treatment of oligometastatic non-small cell lung cancer. J Thorac Dis. 2011; 3(1): 4-9, doi: 10.3978/j.issn.20721439.2010.12.09, indexed in Pubmed: 22263057.

10. Bonnette P. [Non-small cell lung cancer with oligometastases: treatment with curative intent]. Cancer Radiother. 2012; 16(5-6): 344-347, doi: 10.1016/j.canrad.2012.05.017, indexed in Pubmed: 22921976. 